ISSP April No. 2957, 1995, cond-mat/9504102

\title{
Conductivity of 2D lattice electrons in an incommensurate magnetic field
}

\author{
Masahito Takahashi, Yasuhiro Hatsugai ${ }^{*}$ and Mahito Kohmoto \\ Institute for Solid State Physics, University of Tokyo 7-22-1, Roppongi, Minato-ku, Tokyo 106, \\ JAPAN
}

(March, 1995)

\begin{abstract}
We consider conductivities of two-dimensional lattice electrons in a magnetic field. We focus on systems where the flux per plaquette $\phi$ is irrational (incommensurate flux). To realize the system with the incommensurate flux, we consider a series of systems with commensurate fluxes which converge to the irrational value. We have calculated a real part of the longitudinal conductivity $\sigma_{x x}(\omega)$. Using a scaling analysis, we have found $\Re \sigma_{x x}(\omega)$ behaves as $1 / \omega^{\gamma}$ $(\gamma=0.55)$ when $\phi=\tau,\left(\tau=\frac{\sqrt{5}-1}{2}\right)$ and the Fermi energy is near zero. This behavior is closely related to the known scaling behavior of the spectrum.
\end{abstract}

Typeset using REVTEX

*present address: Department of Applied Physics, University of Tokyo, 7-3-1 Hongo, Bunkyo-ku, Tokyo 113, Japan. 


\section{INTRODUCTION}

The electronic properties of the two-dimensional periodic systems in a magnetic field have been studied extensively. Even for non-interacting electrons, the various physical quantities (for example, the wavefunctions, and the energy spectra) exhibit extremely rich behaviors 11 6,8, 15, 17] and it has been attracted great attentions in relation to the quantum Hall effect [7 12], one-dimensional quasiperiodic systems [13 17], flux states for the high- $T_{c}$ superconductivity [18 21]. The algebraic structure of this problem has also been revealed recently 22 24.

Consider the tight-binding Hamiltonian on the square lattice

$$
H^{0}=-\sum_{<i j>} e^{i \theta_{i j}} c_{i}^{\dagger} c_{j}+\text { h.c. }
$$

where $c_{i}^{\dagger}\left(c_{i}\right)$ is a creation (annihilation) operator of an electron at site $i$. The summation is taken over the nearest neighbor sites. The hopping amplitude between link $\langle i j\rangle$ is set to be unity. The phase factor $\theta_{i j}$ is defined on link $\langle i j\rangle$. The magnetic flux per plaquette is $\phi=\frac{1}{2 \pi} \sum_{\text {plaquette }} \theta_{i j}$ in units of magnetic flux quantum $h c / e$.

When $\phi$ is rational, i.e. $\phi=p / q$ with mutual prime integers $p$ and $q$, the spectrum consists of $q$ bands with finite widths. The wave functions are the extended Bloch functions. Many interesting phenomena related to the quantum Hall effects are discussed [8 12].

When $\phi$ is irrational (incommensurate), the system exhibits novel structures [5]. It is known that the electronic state and the energy spectrum have various singular natures. The energy spectrum is a Cartor set which consists of infinitely many bands with zero width [13,15. Especially the system with $\phi=\tau\left(\tau=\frac{\sqrt{5}-1}{2}\right)$ has been extensively studied 15, 17. The spectrum around $E=0$ shows a self-similar structure and the clear scaling behavior is observed. The wavefunctions are critical and some of them show the multifractal behavior [17].

In Ref. [8], it is shown that the Hall conductivity carried is quantized to be an integer in units of $e^{2} / h$ when the Fermi energy $E_{F}$ is in a gap. This integer is given by the first Chern 
number of the fiber bundle on the magnetic Brillouin zone [10]. It is the total vorticity of the $\mathrm{U}(1)$ phase of the Bloch wavefunctions which is a topological invariant. On the other hand, the conductivity of the system is also described by the edge states [11, 12]. It also has a topological origin. The winding number of the edge states in the complex energy plane gives the Hall conductivity [12]. Douçot and Stamp discussed AC conductivities for a commensurate flux $\phi=p / q$ when a particle density $\rho=\phi$ 28]. In this case, the Fermi energy is in the largest gap and the system is an insulator [20,21].

In this paper, we consider both longitudinal and transverse (Hall) conductivity. Especially we are interested in the incommensurate flux limit. When the flux $\phi$ is irrational, there are infinite number of bands with zero width (Cantor set). When the Fermi energy $E_{F}$ is not in a gap with a finite width in the Cantor set, it is highly nontrivial whether the system is metallic or not.

In Sec. [I], we derive the expression for the conductivity $\sigma_{\mu \nu, \phi}(\omega)$ for a commensuarate flux $\phi=p / q$ when $E_{F}$ is at an arbitrary position in the spectrum using the Kubo formula. In the following sections, the longitudinal conductivity is discussed in details. In Sec. III], systems with a sequence of rational numbers $\left\{\phi_{l}\right\}$ are treated numerically in order to understand the system in the incommensurate flux limit. In Sec. $\mathbb{E}$, we discuss the incommensurate limit by taking an appropriate scaling argument. Sec. $\mathrm{V}$ is a summary.

\section{DERIVATION OF THE CONDUCTIVITY}

In this section, we derive an expression for the conductivity $\sigma_{\mu \nu, \phi}(\omega)$ for a rational flux $\phi=p / q$ by the Kubo formula [29]. The real part $\Re \sigma_{x x, \phi}(\omega)$ will be discussed in details.

Let us rewrite the Hamiltonian (1.1) as

$$
\begin{aligned}
H^{0} & =\sum_{\nu=x, y} H_{\nu}^{0}, \\
H_{\nu}^{0} & =-\sum_{\hat{l}}\left(e^{i \theta_{\hat{l}}^{\nu}} c_{\hat{l}+\hat{\nu}}^{\dagger} c_{\hat{l}}+e^{-i \theta_{\hat{l}}^{\nu}} c_{\hat{l}}^{\dagger} c_{\hat{l}+\hat{\nu}}\right) .
\end{aligned}
$$

where $\hat{l}$ denotes a two-dimensional lattice site and $\hat{\nu}$ is a unit vector along the $\nu$ direction. 
The phase factor $\theta_{\hat{l}}^{\nu}$ is on the link between sites $\hat{l}$ and $\hat{l}+\hat{\nu}$. We consider a response of the system to a uniform time-dependent electric field $\boldsymbol{E}(t)=\left(E^{x}(t), E^{y}(t)\right)$. Since the electric field $E_{i j}^{\nu}$ on a link $\langle i j\rangle$ is given by $E_{i j}^{\nu}=-\frac{\hbar}{e} \frac{d \theta_{i j}^{\nu}}{d t}$, the Hamiltonian $H$ with $\boldsymbol{E}(t)$ is given by changing the phase factor $\theta_{\hat{l}}^{\nu}$ in Eq. (2.2) to $\theta_{\hat{l}}^{\nu}+\frac{e}{\hbar} A^{\nu}(t)$ with $-\frac{d A^{\nu}(t)}{d t}=E^{\nu}(t)$. Up to the first order in $\boldsymbol{E}(t)$, the Hamiltonian is expanded as

$$
H=H^{0}+H^{\prime}(t), \quad H^{\prime}(t)=-\sum_{\nu} J_{\nu}^{0} A^{\nu}(t)
$$

where

$$
J_{\mu}^{0}=i \frac{e}{\hbar} \sum_{\hat{l}}\left(e^{i \theta_{\hat{l}}^{\mu}} c_{\hat{l}+\hat{\mu}}^{\dagger} c_{\hat{l}}-e^{-i \theta_{\hat{l}}^{\mu}} c_{\hat{l}}^{\dagger} c_{\hat{l}+\hat{\mu}}\right)
$$

which represents an unperturbed current operator along the $\mu$ direction. The current operator is

$$
J_{\mu}=i \frac{e}{\hbar} \sum_{\hat{l}}\left(e^{i\left(\theta_{\hat{l}}^{\mu}+\frac{e}{\hbar} A^{\mu}(t)\right)} c_{\hat{l}+\hat{\mu}}^{\dagger} c_{\hat{l}}-e^{-i\left(\theta_{\hat{l}}^{\mu}+\frac{e}{\hbar} A^{\mu}(t)\right)} c_{\hat{l}}^{\dagger} c_{\hat{l}+\hat{\mu}}\right) .
$$

It is expanded as

$$
J_{\mu}=J_{\mu}^{0}+\frac{e^{2}}{\hbar^{2}} H_{\mu}^{0} A^{\mu}(t)
$$

up to the first order in $\boldsymbol{E}(t)$. By the Kubo formula, the induced electric current $\delta\left\langle J_{\mu}(t)\right\rangle$ is given by

$$
\begin{aligned}
\delta\left\langle J_{\mu}(t)\right\rangle & =\frac{e^{2}}{\hbar^{2}}\left\langle H_{\mu}^{0}\right\rangle A^{\mu}(t)+\frac{i}{\hbar} \sum_{\nu} \int_{0}^{+\infty} d \tau\left\langle\left[J_{\mu}^{0}(\tau), J_{\nu}^{0}\right]\right\rangle A^{\nu}(t-\tau), \\
J_{\mu}^{0}(\tau) & =e^{i \frac{H^{0}}{\hbar} \tau} J_{\mu}^{0} e^{-i \frac{H^{0}}{\hbar} \tau} .
\end{aligned}
$$

where $\langle\cdots\rangle$ denotes the ground state expectation value. By Fourier transformation, we get

$$
\delta\left\langle J_{\mu}(\omega)\right\rangle=\sum_{\nu}\left[2 \pi i \frac{e^{2}}{h}\left\langle H_{\mu}^{0}\right\rangle \delta_{\mu \nu}-K_{\mu \nu}(\omega)\right] \frac{1}{\hbar \omega-i 0} E^{\nu}(\omega),
$$

with

$$
K_{\mu \nu}(\omega)=\int_{0}^{+\infty} d \tau\left\langle\left[J_{\mu}^{0}(\tau), J_{\nu}^{0}\right]\right\rangle e^{-i \omega \tau}
$$


Let us take the Landau gauge, that is, $\theta_{\hat{l}}^{x}=0, \theta_{\hat{l}}^{y}=2 \pi \phi m$ where $\hat{l}=(m, n)$. In a momentum representation, we have

$$
\begin{aligned}
H^{0} & =\int_{\text {M.B.Z. }} \frac{d^{2} k}{(2 \pi)^{2}} \boldsymbol{c}^{\dagger}(\boldsymbol{k}) \hat{\boldsymbol{H}}^{0}(\boldsymbol{k}) \boldsymbol{c}(\boldsymbol{k}), \\
J_{\mu}^{0} & =\frac{e}{\hbar} \int_{\text {M.B.z. }} \frac{d^{2} k}{(2 \pi)^{2}} \boldsymbol{c}^{\dagger}(\boldsymbol{k}) \hat{\boldsymbol{v}}_{\mu}(\boldsymbol{k}) \boldsymbol{c}(\boldsymbol{k}),
\end{aligned}
$$

with

$$
\begin{aligned}
\hat{\boldsymbol{H}}^{0}(\boldsymbol{k}) & =\sum_{\nu} \hat{\boldsymbol{H}}_{\nu}^{0}(\boldsymbol{k}), \\
\boldsymbol{c}^{\dagger}(\boldsymbol{k}) & =\left[c^{\dagger}\left(k_{x}+2 \pi \phi, k_{y}\right), \cdots, c^{\dagger}\left(k_{x}+2 \pi \phi q, k_{y}\right)\right], \\
\left\{\hat{\boldsymbol{H}}_{x}^{0}(\boldsymbol{k})\right\}_{i, j} & =-2 \cos \left(k_{x}+2 \pi \phi j\right) \delta_{i, j}, \\
\left\{\hat{\boldsymbol{H}}_{y}^{0}(\boldsymbol{k})\right\}_{i, j} & =-\left(e^{-i k_{y}} \delta_{i, j+1}+e^{i k_{y}} \delta_{i, j-1}\right), \\
\left\{\hat{\boldsymbol{v}}_{x}(\boldsymbol{k})\right\}_{i, j} & =2 \sin \left(k_{x}+2 \pi \phi j\right) \delta_{i, j}, \\
\left\{\hat{\boldsymbol{v}}_{y}(\boldsymbol{k})\right\}_{i, j} & =i\left(e^{-i k_{y}} \delta_{i, j+1}-e^{i k_{y}} \delta_{i, j-1}\right), \quad(1 \leq i, j \leq q),
\end{aligned}
$$

where $\boldsymbol{k}=\left(k_{x}, k_{y}\right)$ is defined in the magnetic Brillouin zone (M.B.Z.) $\left(-\pi / q \leq k_{x}<\right.$ $\left.\pi / q,-\pi \leq k_{y}<\pi\right)$, and the operator $c^{\dagger}\left(k_{x}, k_{y}\right)$ is defined by $c^{\dagger}\left(k_{x}, k_{y}\right)=\sum_{m, n} e^{i\left(k_{x} m+k_{y} n\right)} c_{m, n}^{\dagger}$.

Let us discuss a one-particle state of the $l$-th band

$$
\left|\Psi^{l}\right\rangle=\int_{\text {M.B.Z. }} \frac{d^{2} k}{(2 \pi)^{2}} \sum_{j=1}^{q} \psi_{j}^{l}(\boldsymbol{k}) c^{\dagger}\left(k_{x}+2 \pi \phi j, k_{y}\right)|0\rangle .
$$

The Schrödinger equation $H^{0}\left|\Psi^{l}\right\rangle=E^{l}\left|\Psi^{l}\right\rangle$ is reduced to

$$
\hat{\boldsymbol{H}}^{0}(\boldsymbol{k})|\boldsymbol{k}, l\rangle=E^{l}(\boldsymbol{k})|\boldsymbol{k}, l\rangle,
$$

with

$$
|\boldsymbol{k}, l\rangle=\left[\psi_{1}^{l}(\boldsymbol{k}), \cdots, \psi_{q}^{l}(\boldsymbol{k})\right]^{\mathrm{t}}, \quad\left\langle\boldsymbol{k}, l^{\prime} \mid \boldsymbol{k}, l\right\rangle=\delta_{l^{\prime}, l}
$$

From Eqs. (2.12) and (2.10), we have

$$
\begin{aligned}
K_{\mu \nu}(\omega) & =V \frac{e^{2}}{h} \frac{i}{2 \pi} \int_{\text {M.B.Z. }} d^{2} k \sum_{l, l^{\prime}} v_{\mu}^{l l^{\prime}}(\boldsymbol{k}) v_{\nu}^{l^{\prime} l}(\boldsymbol{k}) \frac{f^{l^{\prime}}(\boldsymbol{k})-f^{l}(\boldsymbol{k})}{\hbar \omega-i 0+E^{l^{\prime}}(\boldsymbol{k})-E^{l}(\boldsymbol{k})}, \\
v_{\mu}^{l l^{\prime}}(\boldsymbol{k}) & =\left\langle\boldsymbol{k}, l\left|\hat{\boldsymbol{v}}_{\mu}(\boldsymbol{k})\right| \boldsymbol{k}, l^{\prime}\right\rangle .
\end{aligned}
$$


Similarly we have

$$
\begin{aligned}
\left\langle H_{\mu}^{0}\right\rangle & =V \int_{\text {M.B.Z. }} \frac{d^{2} k}{(2 \pi)^{2}} \sum_{l} \epsilon_{\mu}^{l}(\boldsymbol{k}) f^{l}(\boldsymbol{k}), \\
\epsilon_{\mu}^{l}(\boldsymbol{k}) & =\left\langle\boldsymbol{k}, l\left|\hat{\boldsymbol{H}}_{\mu}^{0}(\boldsymbol{k})\right| \boldsymbol{k}, l\right\rangle,
\end{aligned}
$$

where $V$ is the volume of the system and $f^{l}(\boldsymbol{k})=\theta\left(E_{F}-E^{l}(\boldsymbol{k})\right)$. Since $\delta\left\langle J_{\mu}(\omega)\right\rangle=$ $V \frac{e^{2}}{h} \sum_{\nu} \sigma_{\mu \nu, \phi}(\omega) E^{\nu}(\omega)$, we have

$$
\begin{aligned}
\sigma_{\mu \nu, \phi}(\omega) & =-\frac{1}{2 \pi i} \int_{\text {M.B.Z. }} d^{2} k \sum_{l} \epsilon_{\mu}^{l}(\boldsymbol{k}) f^{l}(\boldsymbol{k}) \frac{1}{\hbar \omega-i 0} \delta_{\mu \nu} \\
& +\frac{1}{2 \pi i} \int_{\text {M.B.Z. }} d^{2} k \sum_{l, l^{\prime}} v_{\mu}^{l l^{\prime}}(\boldsymbol{k}) v_{\nu}^{l^{\prime} l}(\boldsymbol{k}) \frac{f^{l^{\prime}}(\boldsymbol{k})-f^{l}(\boldsymbol{k})}{\hbar \omega-i 0+E^{l^{\prime}}(\boldsymbol{k})-E^{l}(\boldsymbol{k})} \frac{1}{\hbar \omega-i 0} .
\end{aligned}
$$

Let us rewrite the second term as

$$
\begin{gathered}
\frac{1}{2 \pi i} \int_{\text {M.B.Z. }} d^{2} k \sum_{l \neq l^{\prime}} v_{\mu}^{l l^{\prime}}(\boldsymbol{k}) v_{\nu}^{l^{\prime} l}(\boldsymbol{k}) \frac{f^{l^{\prime}}(\boldsymbol{k})-f^{l}(\boldsymbol{k})}{E^{l^{\prime}}(\boldsymbol{k})-E^{l}(\boldsymbol{k})}\left(\frac{1}{\hbar \omega-i 0}-\frac{1}{\hbar \omega-i 0+E^{l^{\prime}}(\boldsymbol{k})-E^{l}(\boldsymbol{k})}\right) \\
=\frac{1}{2 \pi i} \int_{\text {M.B.Z. }} d^{2} k \sum_{l} f^{l}(\boldsymbol{k}) \sum_{l^{\prime} \neq l}\left[v_{\nu}^{l l^{\prime}}(\boldsymbol{k}) \frac{v_{\mu}^{l^{\prime} l}(\boldsymbol{k})}{E^{l}(\boldsymbol{k})-E^{l^{\prime}}(\boldsymbol{k})}-\frac{v_{\mu}^{l l^{\prime}}(\boldsymbol{k})}{E^{l^{\prime}}(\boldsymbol{k})-E^{l}(\boldsymbol{k})} v_{\nu}^{l^{\prime} l}(\boldsymbol{k})\right] \frac{1}{\hbar \omega-i 0} \\
\quad+\frac{1}{2 \pi i} \int_{\text {M.B.Z. }} d^{2} k \sum_{l \neq l^{\prime}} v_{\mu}^{l l^{\prime}}(\boldsymbol{k}) v_{\nu}^{l^{\prime} l}(\boldsymbol{k}) \frac{f^{l^{\prime}}(\boldsymbol{k})-f^{l}(\boldsymbol{k})}{E^{l}(\boldsymbol{k})-E^{l^{\prime}}(\boldsymbol{k})} \frac{1}{\hbar \omega-i 0+E^{l^{\prime}}(\boldsymbol{k})-E^{l}(\boldsymbol{k})} .
\end{gathered}
$$

Using the formulae

$$
\left\langle\boldsymbol{k}, l\left|\frac{\partial}{\partial k_{\mu}}\right| \boldsymbol{k}, l^{\prime}\right\rangle=\frac{v_{\mu}^{l l^{\prime}}(\boldsymbol{k})}{E^{l^{\prime}}(\boldsymbol{k})-E^{l}(\boldsymbol{k})} \quad\left(l \neq l^{\prime}\right),
$$

and

$$
v_{\mu}^{l l}(\boldsymbol{k})=\frac{\partial E^{l}(\boldsymbol{k})}{\partial k_{\mu}},
$$

the first term of Eq. (2.27) is written

$$
\begin{aligned}
& \frac{1}{2 \pi i} \int_{\text {M.B.Z. }} d^{2} k \sum_{l} f^{l}(\boldsymbol{k}) \sum_{l^{\prime} \neq l}\left[v_{\nu}^{l l^{\prime}}(\boldsymbol{k})\left\langle\boldsymbol{k}, l^{\prime}\left|\frac{\partial}{\partial k_{\mu}}\right| \boldsymbol{k}, l\right\rangle-\left\langle\boldsymbol{k}, l\left|\frac{\partial}{\partial k_{\mu}}\right| \boldsymbol{k}, l^{\prime}\right\rangle v_{\nu}^{l^{\prime} l}(\boldsymbol{k})\right] \frac{1}{\hbar \omega-i 0} \\
= & \frac{1}{2 \pi i} \int_{\text {M.B.Z. }} d^{2} k \\
\times & \sum_{l} f^{l}(\boldsymbol{k})\left[\left\langle\boldsymbol{k}, l\left|\hat{\boldsymbol{v}}_{\nu}(\boldsymbol{k}) \frac{\partial}{\partial k_{\mu}}\right| \boldsymbol{k}, l\right\rangle-\langle\boldsymbol{k}, l| \frac{\partial}{\partial k_{\mu}}\left(\hat{\boldsymbol{v}}_{\nu}(\boldsymbol{k})|\boldsymbol{k}, l\rangle\right)+\frac{\partial}{\partial k_{\mu}}\left\langle\boldsymbol{k}, l\left|\hat{\boldsymbol{v}}_{\nu}(\boldsymbol{k})\right| \boldsymbol{k}, l\right\rangle\right] \frac{1}{\hbar \omega-i 0} \\
= & \frac{1}{2 \pi i} \int_{\text {M.B.Z. }} d^{2} k \sum_{l} f^{l}(\boldsymbol{k})\left[-\left\langle\boldsymbol{k}, l\left|\frac{\partial \hat{\boldsymbol{v}}_{\nu}(\boldsymbol{k})}{\partial k_{\mu}}\right| \boldsymbol{k}, l\right\rangle+\frac{\partial v_{\nu}^{l l}(\boldsymbol{k})}{\partial k_{\mu}}\right] \frac{1}{\hbar \omega-i 0} \\
= & \frac{1}{2 \pi i} \int_{\text {M.B.Z. }} d^{2} k \sum_{l} f^{l}(\boldsymbol{k}) \epsilon_{\mu}^{l}(\boldsymbol{k}) \delta_{\mu \nu} \frac{1}{\hbar \omega-i 0}-\frac{1}{2 \pi i} \int_{\text {M.B.Z. }} d^{2} k \sum_{l}\left|v_{\mu}^{l l}(\boldsymbol{k})\right|^{2} \frac{\partial f^{l}(\boldsymbol{k})}{\partial E} \delta_{\mu \nu} \frac{1}{\hbar \omega-i 0} .
\end{aligned}
$$


Next let us separate the $\omega$-dependent part in the second term of Eq. 2.27) and we have

$$
\begin{aligned}
& \frac{1}{2 \pi i} \int_{\text {M.B.Z. }} d^{2} k \sum_{l \neq l^{\prime}} v_{\mu}^{l l^{\prime}}(\boldsymbol{k}) v_{\nu}^{l^{\prime} l}(\boldsymbol{k}) \frac{f^{l^{\prime}}(\boldsymbol{k})-f^{l}(\boldsymbol{k})}{E^{l}(\boldsymbol{k})-E^{l^{\prime}}(\boldsymbol{k})} \frac{1}{\hbar \omega-i 0+E^{l^{\prime}}(\boldsymbol{k})-E^{l}(\boldsymbol{k})} \\
= & \frac{1}{2 \pi i} \int_{\text {M.B.Z. }} d^{2} k \sum_{l \neq l^{\prime}} v_{\mu}^{l l^{\prime}}(\boldsymbol{k}) v_{\nu}^{l^{\prime} l}(\boldsymbol{k}) \frac{f^{l}(\boldsymbol{k})-f^{l^{\prime}}(\boldsymbol{k})}{\left(E^{l}(\boldsymbol{k})-E^{l^{\prime}}(\boldsymbol{k})\right)^{2}} \\
& +\frac{1}{2 \pi i} \int_{\text {M.B.Z. }} d^{2} k \sum_{l \neq l^{\prime}} v_{\mu}^{l l^{\prime}}(\boldsymbol{k}) v_{\nu}^{l^{\prime} l}(\boldsymbol{k}) \frac{f^{l^{\prime}}(\boldsymbol{k})-f^{l}(\boldsymbol{k})}{\left(E^{l}(\boldsymbol{k})-E^{l^{\prime}}(\boldsymbol{k})\right)^{2}} \frac{\hbar \omega}{\hbar \omega-i 0+E^{l^{\prime}}(\boldsymbol{k})-E^{l}(\boldsymbol{k})},
\end{aligned}
$$

Using Eq. (2.28) and $\left\langle\boldsymbol{k}, l\left|\frac{\partial}{\partial k_{\nu}}\right| \boldsymbol{k}, l^{\prime}\right\rangle=-\left(\frac{\partial}{\partial k_{\nu}}\langle\boldsymbol{k}, l|\right)\left|\boldsymbol{k}, l^{\prime}\right\rangle$, the first term of Eq. 2.31) is written

$$
\begin{aligned}
& \frac{1}{2 \pi i} \int_{\text {M.B.Z. }} d^{2} k \sum_{l} f^{l}(\boldsymbol{k}) \sum_{l^{\prime} \neq l}\left[\frac{v_{\mu}^{l l^{\prime}}(\boldsymbol{k}) v_{\nu}^{l^{\prime} l}(\boldsymbol{k})-v_{\nu}^{l l^{\prime}}(\boldsymbol{k}) v_{\mu}^{l^{\prime} l}(\boldsymbol{k})}{\left(E^{l}(\boldsymbol{k})-E^{l^{\prime}}(\boldsymbol{k})\right)^{2}}\right] \\
= & \frac{1}{2 \pi i} \int_{\text {M.B.Z. }} d^{2} k \sum_{l} f^{l}(\boldsymbol{k}) \sum_{l^{\prime}}\left[\left\langle\boldsymbol{k}, l\left|\frac{\partial}{\partial k_{\nu}}\right| \boldsymbol{k}, l^{\prime}\right\rangle\left\langle\boldsymbol{k}, l^{\prime}\left|\frac{\partial}{\partial k_{\mu}}\right| \boldsymbol{k}, l\right\rangle-\left\langle\boldsymbol{k}, l\left|\frac{\partial}{\partial k_{\mu}}\right| \boldsymbol{k}, l^{\prime}\right\rangle\left\langle\boldsymbol{k}, l^{\prime}\left|\frac{\partial}{\partial k_{\nu}}\right| \boldsymbol{k}, l\right\rangle\right] \\
= & \frac{1}{2 \pi i} \int_{\text {M.B.Z. }} d^{2} k \sum_{l} f^{l}(\boldsymbol{k})\left[\left(\frac{\partial}{\partial k_{\mu}}\langle\boldsymbol{k}, l|\right) \frac{\partial}{\partial k_{\nu}}|\boldsymbol{k}, l\rangle-\left(\frac{\partial}{\partial k_{\nu}}\langle\boldsymbol{k}, l|\right) \frac{\partial}{\partial k_{\mu}}|\boldsymbol{k}, l\rangle\right] \\
= & \frac{1}{2 \pi i} \int_{\text {M.B.Z. }} d^{2} k \sum_{l} f^{l}(\boldsymbol{k}) \nabla \times\langle\boldsymbol{k}, l|\nabla| \boldsymbol{k}, l\rangle \epsilon_{\mu \nu}
\end{aligned}
$$

From Eqs. (2.26), (2.30), (2.31) and (2.32) with $\partial f^{l}(\boldsymbol{k}) / \partial E=-\delta\left(E_{F}-E^{l}(\boldsymbol{k})\right)$, we have

$$
\begin{aligned}
\sigma_{\mu \nu, \phi}(\omega)= & \frac{1}{2 \pi i} \int_{\text {M.B.Z. }} d^{2} k \sum_{l}\left|v_{\mu}^{l l}(\boldsymbol{k})\right|^{2} \delta\left(E_{F}-E^{l}(\boldsymbol{k})\right) \frac{1}{\hbar \omega-i 0} \delta_{\mu \nu} \\
& +\frac{1}{2 \pi i} \int_{\text {M.B.Z. }} d^{2} k \sum_{l} f^{l}(\boldsymbol{k}) \nabla \times\langle\boldsymbol{k}, l|\nabla| \boldsymbol{k}, l\rangle \epsilon_{\mu \nu} \\
& +\frac{1}{2 \pi i} \int_{\text {M.B.Z. }} d^{2} k \sum_{l \neq l^{\prime}} v_{\mu}^{l l^{\prime}}(\boldsymbol{k}) v_{\nu}^{l^{\prime} l}(\boldsymbol{k}) \frac{f^{l^{\prime}}(\boldsymbol{k})-f^{l}(\boldsymbol{k})}{\left(E^{l}(\boldsymbol{k})-E^{l^{\prime}}(\boldsymbol{k})\right)^{2}} \frac{\hbar \omega}{\hbar \omega-i 0+E^{l^{\prime}}(\boldsymbol{k})-E^{l}(\boldsymbol{k})} .
\end{aligned}
$$

For the Hall conductivity $\sigma_{x y, \phi}(\omega)$, we have

$$
\begin{aligned}
\sigma_{x y, \phi}(\omega) & =\sigma_{x y, \phi}^{\mathrm{TKNN}}+\sigma_{x y, \phi}^{S}(\omega), \\
\sigma_{x y, \phi}^{\mathrm{TKNN}} & =\frac{1}{2 \pi i} \int_{\text {M.B.Z. }} d^{2} k \sum_{l} f^{l}(\boldsymbol{k}) \nabla \times\langle\boldsymbol{k}, l|\nabla| \boldsymbol{k}, l\rangle, \\
\sigma_{x y, \phi}^{S}(\omega) & =\frac{1}{2 \pi i} \int_{\text {M.B.Z. }} d^{2} k \sum_{l \neq l^{\prime}} v_{x}^{l l^{\prime}}(\boldsymbol{k}) v_{y}^{l^{\prime} l}(\boldsymbol{k}) \frac{f^{l^{\prime}}(\boldsymbol{k})-f^{l}(\boldsymbol{k})}{\left(E^{l}(\boldsymbol{k})-E^{l^{\prime}}(\boldsymbol{k})\right)^{2}} \frac{\hbar \omega}{\hbar \omega-i 0+E^{l^{\prime}}(\boldsymbol{k})-E^{l}(\boldsymbol{k})} .
\end{aligned}
$$

Since $\sigma_{x y, \phi}^{S}(\omega=0)=0$, the static Hall conductivity is given by $\sigma_{x y, \phi}^{\mathrm{TKNN}}$ which is a topological invariant in the M.B.Z. 
For the longitudinal conductivity $\sigma_{x x, \phi}(\omega)$, we have

$$
\begin{aligned}
\sigma_{x x, \phi}(\omega) & =\sigma_{x x, \phi}^{D}(\omega)+\sigma_{x x, \phi}^{S}(\omega), \\
\sigma_{x x, \phi}^{D}(\omega) & =\frac{1}{2 \pi i} \int_{\text {M.B.z. }} d^{2} k \sum_{l}\left|v_{x}^{l l}(\boldsymbol{k})\right|^{2} \delta\left(E_{F}-E^{l}(\boldsymbol{k})\right) \frac{1}{\hbar \omega-i 0}, \\
\sigma_{x x, \phi}^{S}(\omega) & =\frac{1}{2 \pi i} \int_{\text {M.B.z. }} d^{2} k \sum_{l \neq l^{\prime}}\left|v_{x}^{l l^{\prime}}(\boldsymbol{k})\right|^{2} \frac{f^{l^{\prime}}(\boldsymbol{k})-f^{l}(\boldsymbol{k})}{\left(E^{l}(\boldsymbol{k})-E^{l^{\prime}}(\boldsymbol{k})\right)^{2}} \frac{\hbar \omega}{\hbar \omega-i 0+E^{l^{\prime}}(\boldsymbol{k})-E^{l}(\boldsymbol{k})},
\end{aligned}
$$

where $\sigma_{x x, \phi}^{D}(\omega)$ is the so-called Drude term and $\sigma_{x x, \phi}^{S}(\omega)$ is the contribution from interband scattering processes. Real parts of them are evaluated as

$$
\begin{aligned}
\Re \sigma_{x x, \phi}^{D}(\omega) & =D(q) \delta(\hbar \omega), \\
D(q) & =\frac{1}{2} \int_{\text {M.B.z. }} d^{2} k \sum_{l}\left|v_{x}^{l l}(\boldsymbol{k})\right|^{2} \delta\left(E_{F}-E^{l}(\boldsymbol{k})\right), \\
& = \begin{cases}\frac{1}{2} \oint_{E^{m}(k)=E_{F}} d k\left|v_{x}^{m m}(\boldsymbol{k})\right|^{2} \frac{1}{\left|\nabla E^{m}(k)\right|} & \left(E_{F} \text { is in the } m \text {-th band }\right) \\
0 & \left(E_{F} \text { is in the energy gap }\right),\end{cases}
\end{aligned}
$$

and

$$
\begin{aligned}
\Re \sigma_{x x, \phi}^{S}(\omega) & =\frac{1}{2} \int_{\text {M.B.Z. }} d^{2} k \sum_{l \neq l^{\prime}}\left|v_{x}^{l l^{\prime}}(\boldsymbol{k})\right|^{2}\left\{f^{l^{\prime}}(\boldsymbol{k})-f^{l}(\boldsymbol{k})\right\} \delta\left(\hbar \omega+E^{l^{\prime}}(\boldsymbol{k})-E^{l}(\boldsymbol{k})\right) \frac{1}{\hbar \omega} \\
& =\frac{1}{2 \hbar \omega} \sum_{l \neq l^{\prime}, E^{l} \geq E_{F} \geq E^{l^{\prime}}} \oint_{E^{l}(k)-E^{l^{\prime}}(k)=\hbar \omega} d k\left|v_{x}^{l l^{\prime}}(\boldsymbol{k})\right|^{2} \frac{1}{\left|\nabla\left(E^{l}(k)-E^{l^{\prime}}(k)\right)\right|}
\end{aligned}
$$

The Drude term $\Re \sigma_{x x, \phi}^{D}(\omega)$ is a delta function at $\omega=0$ with the weight being determined by the average velocity of the electrons at the Fermi lines (there are $q$ Fermi lines). The second term $\Re \sigma_{x x, \phi}^{S}(\omega)$ comes from the interband scattering processes between states $\left(\boldsymbol{k}, l^{\prime}\right)$ and $(\boldsymbol{k}, l)$.

For a rational flux, the onset of $\Re \sigma_{x x, \phi}^{S}(\omega)$ is given by the minimum energy gap. When the Fermi energy is in the energy band, the Drude term $\Re \sigma_{x x, \phi}^{D}(\omega)$ can also contribute.

\section{NUMERICAL RESULTS FOR THE IRRATIONAL FLUX}

In order to understand the incommensurate flux case, we approximate the irrational $\phi$ by a series of rational fluxes which tends to the irrational as $q \rightarrow \infty$. For a large $q$, the onset 
of the $\Re \sigma_{x x, \phi}^{S}(\omega)$ becomes small and it is difficult to distinguish $\Re \sigma_{x x, \phi}^{S}(\omega)$ from $\Re \sigma_{x x, \phi}^{D}(\omega)$. Thus careful considerations are necessary to understand the behavior of $\Re \sigma_{x x, \phi}(\omega)$.

Let us consider the systems with $\phi_{l}=F_{l} / F_{l+1}$ where $F_{l}$ is the Fibonacci number defined by $F_{l+1}=F_{l}+F_{l-1}$ with $F_{0}=F_{1}=1$. The flux $\phi_{l}$ tends to $\phi=\frac{\sqrt{5}-1}{2}$ (the inverse of the golden mean for $l \rightarrow \infty)$. Calculations were done for $l=5 \sim 12$ which correspond to the fluxes $\frac{8}{13}, \frac{13}{21}, \frac{21}{34}, \frac{34}{55}, \frac{55}{89}, \frac{89}{144}, \frac{144}{233}$ and $\frac{233}{377}$. The one-dimensional integrations in Eq. (2.41) and Eq. (2.42) were numerically performed by the double exponential formula.

First $E_{F}$ was put in an energy band. We kept $E_{F}$ near zero since there is a energy band around $E=0$ if $q$ is odd. The cases with even $q$ were avoided since the density of states disappear linearly at $E=0$ [25] 27]. The Drude weights $D(q)$ are shown in Fig.1. We treated three systems with $E_{F}=0, E_{F}=-0.475 \Delta_{l}$ and $E_{F}=-0.4995 \Delta_{l}$ where $\Delta_{l}$ is a width of the central band in the $l$-th stage. In some cases, we have data for $\phi=610 / 987(l=14)$. Since it is known that the spectrum shows a self-similar structure for every three fluxes $\left(\phi_{l}, \phi_{l+3}, \cdots\right)$ [15], we fitted the results within these groups. Numerical results are well fitted by the following scaling form

$$
D\left(q_{l}\right)=\bar{D} \frac{1}{q_{l}^{\alpha}}, \quad \alpha=0.828 \pm 0.002 .
$$

where the constant $\bar{D}$ depends on the position of $E_{F}$ but $\alpha$ is universal (see Fig.1). We calculated the interband scattering term $\Re \sigma_{x x, \phi_{l}}^{S}(\omega)$ when $E_{F}=0$ and they are shown in Figs.2. In the $q_{l} \rightarrow \infty$ limit, the energy gap near $E=0$ becomes infinitesimally small and the interband scattering term $\Re \sigma_{x x, \phi_{l}}^{S}(\omega)$ can also contribute in $\omega \sim 0$. Next let us put $E_{F}$ in the energy gap near $E=0$. We have calculated $\Re \sigma_{x x, \phi_{l}}^{S}(\omega)$ when $E_{F}$ is in the gap just above $E=0$. Numerical calculations for even $q$ are also included. In this case, the Drude term does not contribute and we have calculated only $\Re \sigma_{x x, \phi_{l}}^{S}(\omega)$. Numerical results are shown in Figs.3.

As shown in Figs.2 and Figs.3, the self-similar structure is reflected on the $\omega$-dependence of $\Re \sigma_{x x, \phi_{l}}^{S}(\omega)$. This is consistent with the the self-similar behavior observed in the energy spectrum. The supports of $\Re \sigma_{x x, \phi_{l}}^{S}(\omega)$ vanish when the flux approaches to the irrational 
value.

\section{DISCUSSIONS}

Since $\Re \sigma_{x x, \phi_{l}}(\omega)$ shows the complex behavior, we analyze the numerical results in Sec. III carefully and discuss scaling behaviors of the longitudinal conductivity. Since $\phi_{l}$ can be considered as $l$-th approximation of the irrational flux $\phi=\tau$, the energy resolution of the calculations should be determined by the spectrum in the $l$-th approximation. Thus $\Re \sigma_{x x, \phi_{l}}^{(D, S)}(\omega)$ is averaged over the energy window $\left[\omega-W_{l} / 2, \omega+W_{l} / 2\right]$ to define $\Re \sigma_{\text {ave, } \phi_{l}}^{(D, S)}(\omega)$

$$
\Re \sigma_{\mathrm{ave}, \phi_{l}}^{(D, S)}(\omega)=\frac{1}{W_{l}} \int_{\omega-W_{l} / 2}^{\omega+W_{l} / 2} \Re \sigma_{x x, \phi_{l}}^{(D, S)}\left(\omega^{\prime}\right) d \omega^{\prime},
$$

where $W_{l}$ is determined by the energy resolution of the energy spectrum. We put $\omega=0$

to consider the small $\omega$ behavior. Denote $\Re \sigma_{\text {ave, } \phi_{l}}^{(D, S)}(0)$ by $\Re \sigma_{\text {ave }}^{(D, S)}\left(\phi_{l}\right)$. We take $W_{l}$ the same as the energy scale $\Delta E_{l}$ of the energy spectrum around $E=0$. Let us take $\Delta E_{l}$ to be the energy gap around $E=0$. The scaling form of $\Delta E_{l}$ is numerically given by

$$
\Delta E_{l}=\frac{\text { const. }}{q_{l}^{\eta}}, \quad \eta=1.8285 \pm 0.0006
$$

This scaling behavior was already discussed in Ref. [15]. We choose the window $W_{l}$ as

$$
W_{l}=\frac{W_{0}}{q_{l}^{\eta}}
$$

where $W_{0}$ is a $q$-independent constant. The number of bands $M$ within the window $W_{l}$ is determined by $W_{0}$ which is independent of $l$. The average conductivities $\Re \sigma_{\text {ave }}\left(\phi_{l}\right)$ were calculated for three values of $W_{0}$ which give $M=3,7$, and 11 respectively. We consider the two cases separately. One is when the Fermi energy is in the energy gap and the other is when the Fermi energy is in the energy band. First let us consider the former case. The averaged values of $\Re \sigma_{\text {ave }}^{S}\left(\phi_{l}\right)$ are shown in Fig.4.(a). The results suggest strongly the scaling behavior

$$
\Re \sigma_{\text {ave }}^{S}\left(\phi_{l}\right)=c\left(W_{0}\right) q_{l}^{\delta_{S}}, \quad \delta_{S}=0.99 \pm 0.02
$$


The coefficient $c\left(W_{0}\right)$ depends on the system but it is independent of $q_{l}$. Next let us consider the latter case when $E_{F}$ is in the band. We calculated $\Re \sigma_{\text {ave }}^{S}\left(\phi_{l}\right)$ similarly. The results are shown in Fig.4.(b). They also suggest the scaling form Eq. (4.4) with the exponent $\delta_{S}=0.99 \pm 0.07$. In this case, the Drude term $\Re \sigma_{x x, \phi_{l}}^{D}(\omega)=D(q) \delta(\hbar \omega)$ also contributes to $\Re \sigma_{\text {ave }}\left(\phi_{l}\right)$ since the Fermi energy is in the energy band. The contribution from the Drude term is obtained as

$$
\Re \sigma_{\text {ave }}^{D}\left(\phi_{l}\right)=\frac{1}{W_{l}} \int_{-W_{l} / 2}^{W_{l} / 2} \Re \sigma_{x x, \phi_{l}}^{D}\left(\omega^{\prime}\right) d \omega^{\prime}=c^{\prime}\left(W_{0}\right) q_{l}^{\delta_{D}}
$$

where $c^{\prime}\left(W_{0}\right)$ is independent of $q_{l}$. The scaling exponent $\alpha$ for the Drude weight is defined from Eqs. (4.3) and (4.5) to be

$$
\alpha=\eta-\delta_{D}
$$

The value of the exponent $\delta_{D}$ is identical to $\delta_{S}$ within our numerical accuracy.

Define $\delta \equiv \delta_{S} \simeq \delta_{D}$, then the average conductivity $\Re \sigma_{\text {ave }}\left(\phi_{l}\right)$ has the scaling form

$$
\Re \sigma_{\text {ave }}\left(\phi_{l}\right)=c\left(W_{0}\right) q_{l}^{\delta}
$$

This form is universal in the sense that the exponent $\delta$ is independent of the position of $E_{F}$ and the way the approximation of the irrational flux is made. Furthermore, it does not depend on whether the Fermi energy is in an energy gap or not. From Eqs. (4.2) and (4.7), we have the scaling

$$
\Re \sigma_{x x, \phi}(\omega)=\frac{\text { const. }}{\omega^{\gamma}}, \quad \text { for } \omega \rightarrow 0
$$

where

$$
\gamma=\frac{\delta}{\eta} \simeq 0.55
$$

The naive argument would lead to $\Re \sigma_{x x, \phi}(\omega) \simeq c \delta(\omega)+c^{\prime} / \omega$ when $E_{F}$ is in the energy band (metal) and $\Re \sigma_{x x, \phi}(\omega) \simeq c^{\prime} / \omega$ when $E_{F}$ is in the energy gap (insulator) as $\omega$ tends to zero. Our result Eq. (4.8) is different from both of these simple expectations. We shall 
give the correct analysis below. Let us assume that the matrix elements in Eqs. (2.41) and (2.42) to be constant $\left|v_{x}^{l l^{\prime}}(\boldsymbol{k})\right|=v_{x}$. Then the Drude weight $D(q)$ can be estimated as

$$
D(q) \simeq \frac{1}{2} v_{x}^{2} \oint_{E^{m}\left(k_{x}, k_{y}\right)=E_{F}} d k \frac{1}{\left|\nabla E^{m}(\boldsymbol{k})\right|}=2 \pi^{2} N^{m}\left(E_{F}\right) v_{x}^{2}
$$

where $N^{m}(E)=\frac{1}{(2 \pi)^{2}} \oint_{E^{m}\left(k_{x}, k_{y}\right)=E} d k \frac{1}{\left|\nabla E^{m}\right|}$ is a density of states of the $m$-th band. Since there are $q$ bands in the total spectrum, integrated density of states for each band is $1 / q$ and $N\left(E_{F}\right) \sim \frac{1}{q \Delta E}$, where $\Delta E$, the width of the band including $E=0$, is of the order of $\Delta E \sim q^{-\eta}$ (see Eq. (4.2)). Also we may estimate $v_{x}=\Delta E / \Delta k_{x}$ where $\Delta k_{x} \sim 1 / q$ is a size of the magnetic Brillouin zone in the $x$ direction. Thus we have

$$
D(q) \simeq \text { const. } \times q^{1-\eta}
$$

It suggests a scaling relation

$$
\alpha=\eta-1
$$

which is consistent with the numerical result of $\alpha=0.828 \pm 0.002$ and $\eta=1.8285 \pm 0.0006$. It gives the scaling relation between the Drude weight and the spectrum. Note the scaling index for the Drude weight is $\alpha$ and the scaling index for the spectrum is $\eta$. From Eq. (4.6) it implies

$$
\delta_{D}=1
$$

Next let us consider the contribution from the interband scattering Eq. (2.42). Since the onset of $\Re \sigma_{x x, \phi_{l}}^{S}(\omega)$ is of the order of $\Delta E$, we have

$$
\Re \sigma_{\mathrm{ave}}^{S}\left(\phi_{l}\right) \simeq \frac{1}{\Delta E} v_{x}^{2} N_{F} N_{c}
$$

where $N_{c}(\sim O(1))$ is a number of a possible combination of the energy bands which can contribute the process. Thus we can estimate $\Re \sigma_{\text {ave }}^{S}\left(\phi_{l}\right)$ as $\Re \sigma_{\text {ave }}^{S}\left(\phi_{l}\right) \simeq \frac{1}{\Delta E}(q \Delta E)^{2} \frac{1}{q \Delta E}=q$. It suggests

$$
\delta_{S}=1
$$




\section{SUMMARY}

We have considered both of the longitudinal and transverse conductivities $\sigma_{\mu \nu, \phi}(\omega)$ of the two-dimensional electrons on the square lattice at an arbitrary filling factor. Especially the longitudinal conductivity $\sigma_{x x, \phi}(\omega)$ is discussed in details. It consists of two terms: the Drude term $\sigma_{x x, \phi}^{D}(\omega)$ and the interband scattering term $\sigma_{x x, \phi}^{S}(\omega)$.

We numerically calculated the real parts $\Re \sigma_{x x, \phi_{l}}^{D}(\omega)$ and $\Re \sigma_{x x, \phi_{l}}^{S}(\omega)$ for a series of sys-

tems with rational fluxes $\left\{\phi_{l}\right\}$ which converge to the irrational value $\tau=\frac{\sqrt{5}-1}{2}$. The case when the Fermi energy $E_{F}$ lies near $E=0$ was treated in details. Two parts of the longitudinal conductivities $\Re \sigma_{x x, \phi_{l}}^{D}(\omega)$ and $\Re \sigma_{x x, \phi_{l}}^{S}(\omega)$ show the same scaling behavior in the incommensurate limit. Taking into account the self-similar structure of the energy spectrum, we performed the averaging procedure near $\omega=0$. It revealed the clear scaling behavior $\Re \sigma_{x x}(\omega) \sim 1 / \omega^{\gamma}(\gamma \simeq 0.55, \omega \rightarrow 0)$. This result is quite distinct from the commensurate case. 


\section{REFERENCES}

$\dagger \quad$ electronic address: hatsugai@tansei.cc.u-tokyo.ac.jp

[1] P. G. Harper, Proc. Phys. Soc. Lond. A68 (1955) 874.

[2] G. H. Wannier, Rev. Mod. Phys. 34 (1962) 645

[3] M. Ya. Azbel, Soviet Phys. JETP 19 (1964) 634

[4] J. Zak, Phys. Rev. 134 (1964) A1602

[5] D. R. Hofstadter, Phys. Rev. B14 (1976) 2239

[6] G. H. Wannier, G. M. Obermair, and R. Ray, Phys. Stat. Sol. 93 (1979) 337

[7] The Quantum Hall Effect, edited by R. E. Prange and S. M. Girvin (Springer-Verlag, Berlin, 1987)

[8] D. J. Thouless, M. Kohmoto, M. P. Nightingale, and M. den Nijs, Phys. Rev. Lett 49 (1982) 405

[9] J. Avron, R. Seiler, and B. Simon, Phys. Rev. Lett. 51 (1983) 51

[10] M. Kohmoto, Ann. Phys. (N.Y.) 160 (1985) 355

[11] R. Rammal, G. Toulouse, M. T. Jaekel, and B. I. Halperin, Phys. Rev. B27 (1983) 5142

[12] Y. Hatsugai, Phys. Rev. Lett. 71 (1993) 3697, Phys. Rev. B48 (1993) 11851

[13] S. Aubry and G. André, Ann. Isr. Phys. Soc. 3 (1980) 133

[14] D. J. Thouless, Phys. Rev. B28 (1983) 4272

[15] M. Kohmoto, Phys. Rev. Lett. 51 (1983) 1198

[16] S. Ostlund and R. Pandit, Phys. Rev. B29 (1984) 1394

[17] H. Hiramoto and M. Kohmoto, Int. Jour. Mod. Phys. B 6 (1992) 281-320 
[18] X. G. Wen, F. Wilczek, and A. Zee, Phys. Rev. B39 (1989) 11413

[19] I. Affleck, Z. Zou, T. Hsu, and P. W. Anderson, Phys. Rev. B38 (1988) 745

[20] Y. Hasegawa, P. Lederer, T. M. Rice, and P. B. Wiegmann, Phys. Rev. Lett. 63 (1989) 907

[21] Y. Hasegawa, Y. Hatsugai, M. Kohmoto, and G. Montambaux, Phys. Rev. B41 (1990) 9174

[22] P. B. Wiegmann and A. V. Zabrodin, Phys. Rev. Lett. 72 (1944) 1890

[23] Y. Hatsugai, M. Kohmoto, and Y. S. Wu, Phys. Rev. Lett. 73 (1994) 1134

[24] L. D. Fadeev and R. M. Kashaev, University of Helsinky Report No. HU-TFT-93-63, December 1993

[25] X. G. Wen and A. Zee, Nucl. Phys. B316 (1989) 641

[26] M. Kohmoto, Phys. Rev. B39 (1989) 11943

[27] Y. Hatsugai and M. Kohmoto, Phys. Rev. B42 (1990) 8282

[28] B. Douçot and P. C. E. Stamp, Phys. Rev. Lett. 66 (1991) 2503

[29] R. Kubo, S. J. Miyake, and N. Hashitsume, Solid State Physics, 17 (1965) 269 (ed. F. Seitz, D. Turnbull, and H. Ehrenreich, Academic Press) 


\section{FIGURES}

FIG. 1. Drude weights $D(q)$ for systems with three Fermi energies $E_{F}$ in the central band; (a) $E_{F}=0, \phi=\frac{3}{5}, \frac{13}{21}, \frac{55}{89}$, and $\frac{233}{377}$, (b) $E_{F}=0, \phi=\frac{8}{13}, \frac{34}{55}, \frac{144}{233}$, and $\frac{610}{987}$, (c) $E_{F}=-0.475 \Delta_{l}, \phi=\frac{3}{5}$, $\frac{13}{21}, \frac{55}{89}$, and $\frac{233}{377}$, (d) $E_{F}=-0.475 \Delta_{l}, \phi=\frac{8}{13}, \frac{34}{55}, \frac{144}{233}$, and $\frac{610}{987},(\mathrm{e}) E_{F}=-0.4995 \Delta_{l}, \phi=\frac{3}{5}, \frac{13}{21}$, $\frac{55}{89}$, and $\frac{233}{377}$, and (f) $E_{F}=-0.4995 \Delta_{l}, \phi=\frac{8}{13}, \frac{34}{55}, \frac{144}{233}$, and $\frac{610}{987}$ where $\Delta_{l}$ is a width of the central band.

FIG. 2. $\Re \sigma_{x x, \phi_{l}}^{S}(\omega)$ for systems with rational fluxes $\left\{\phi_{l}\right\}$ with $E_{F}=0$; (a) $\phi_{5}=8 / 13$, (b) $\phi_{6}=13 / 21$, (c) $\phi_{8}=34 / 55$, (d) $\phi_{9}=55 / 89$, (e) $\phi_{11}=144 / 233$ and (f) $\phi_{12}=233 / 377$. Note the differences in the energy scales.

FIG. 3. $\Re \sigma_{x x, \phi_{l}}^{S}(\omega)$ for systems with rational fluxes $\left\{\phi_{l}\right\}$; (a) $\phi_{5}=8 / 13$, (b) $\phi_{6}=13 / 21$, (c) $\phi_{7}=21 / 34,(\mathrm{~d}) \phi_{8}=34 / 55$, (e) $\phi_{9}=55 / 89$, (f) $\phi_{10}=89 / 144,(\mathrm{~g}) \phi_{11}=144 / 233$ and $(\mathrm{h})$ $\phi_{12}=233 / 377$. The Fermi energy $E_{F}$ for each of them lies in the gap just above $E=0$. Note the differences in the energy scales.

FIG. 4. Averaged conductivities $\Re \sigma_{\text {ave }}^{S}\left(\phi_{l}\right)$ in two cases; (a) when $E_{F}$ is in the energy gap, and (b) when $E_{F}$ is in the energy band. $\Re \sigma_{\text {ave }}^{S}\left(\phi_{l}\right)$ was calculated for three values of $W_{0}$ which give $M=3,7$, and 11 , respectively; (a) (1) $M=3, \phi=\frac{8}{13}, \frac{34}{55}, \frac{144}{233},(2) M=3, \phi=\frac{13}{21}, \frac{55}{89}, \frac{233}{377}$, (3) $M=3, \phi=\frac{21}{34}, \frac{89}{144},(4) M=7, \phi=\frac{8}{13}, \frac{34}{55}, \frac{144}{233},(5) M=7, \phi=\frac{13}{21}, \frac{55}{89}, \frac{233}{377},(6) M=7, \phi=\frac{21}{34}$, $\frac{89}{144},(7) M=11, \phi=\frac{8}{13}, \frac{34}{55}, \frac{144}{233},(8) M=11, \phi=\frac{13}{21}, \frac{55}{89}, \frac{233}{377}$, and $(9) M=11, \phi=\frac{21}{34}, \frac{89}{144}$, and (b) (1) $M=3, \phi=\frac{8}{13}, \frac{34}{55}, \frac{144}{233},(2) M=3, \phi=\frac{13}{21}, \frac{55}{89}, \frac{233}{377},(3) M=7, \phi=\frac{8}{13}, \frac{34}{55}, \frac{144}{233},(4) M=7$, $\phi=\frac{13}{21}, \frac{55}{89}, \frac{233}{377},(5) M=11, \phi=\frac{8}{13}, \frac{34}{55}, \frac{144}{233}$, and $(6) M=11, \phi=\frac{13}{21}, \frac{55}{89}, \frac{233}{377}$. 